\title{
Three-Dimensional Massive Gauge Theories
}

\author{
S. Deser \\ Department of Physics, Brandeis University, Waltham, Massachusetts 02254 \\ and \\ R. Jackiw and S. Templeton \\ Center for Theoretical Physics, Laboratory for Nuclear Science and Department of Physics, \\ Massachusetts Institute of Technology, Cambridge, Massachusetts 02139 \\ (Received 22 January 1982)

\begin{abstract}
Three-dimensional Yang-Mills and gravity theories augmented by gauge-invariant mass terms are analyzed. These topologically nontrivial additions profoundly alter the particle content of the models and lead to quantization of a dimensionless mass-coupling-constant ratio. The vector field excitations become massive, with spin 1 (rather than massless with spin 0), and the mass provides an infrared cutoff. The gravitation acquires mass, mediates finite-range interactions, and has spin 2 (rather than being absent altogether); although its mass term is of third derivative order, there are no ghosts or acausalities.
\end{abstract}

PACS numbers: 11.15.- $\mathrm{z}, 04.60 .+\mathrm{n}, 11.10 . \mathrm{Gh}$

The topology of odd-dimensional spaces permits the construction of gauge theories with novel and attractive properties. We treat here the non-Abelian vector and tensor models in three space-time dimensions. ${ }^{1,2}$ In both, a topological structure, the Chern-Simons secondary characteristic, ${ }^{3}$ augments the usual action. These gauge-invariant terms dramatically affect the nature of the field excitations, changing their spin and endowing them with a mass. For the (superrenormalizable) vector model, the spin changes from 0 to 1 and the mass parameter provides a perturbative inf rared cutoff. In the gravitational case, the dynamically trivial Einstein action becomes that of a finite-range spin-2 field; despite the third derivative order of the mass term, there are neither ghosts nor acausalities. The non-Abelian mass term also has nontrivial homotopy properties, which lead to quantization of a dimensionless combination of mass and coupling constant.

The vector field action is

$$
I_{V}=I_{\mathrm{YM}}+I_{V C S}=\left(g^{-2} / 2\right) \int d^{3} x \operatorname{tr} F_{\alpha \beta} F^{\alpha \beta}-\left(\mu g^{-2} / 2\right) \int d^{3} x \epsilon^{\alpha \beta} \gamma \operatorname{tr}\left[F_{\alpha \beta} A_{\gamma}-\frac{2}{3} A_{\alpha} A_{\beta} A_{\gamma}\right]
$$

in the usual matrix notation for the internal indices. The gravity action reads

$$
I_{G}=I_{\mathrm{E}}+I_{G C S}=\kappa^{-2} \int d^{3} x g^{1 / 2} R-\left(4 \mu \kappa^{2}\right)^{-1} \int d^{3} x \epsilon^{\alpha \beta \gamma}\left(R_{\alpha \beta a b} \omega_{\gamma}{ }^{a b}+\frac{2}{3} \omega_{\alpha a b} \omega_{\beta}{ }^{b c} \omega_{\gamma c}{ }^{a}\right) \text {. }
$$

Note the sign of the Einstein term, which is opposite to that in four-dimensional gravity. The coupling constants $(g, \kappa)$ have dimension (mass) ${ }^{1 / 2}$, and $\mu$ is the mass parameter; our signature is $(+--)$. In (1b), $\omega_{\alpha a b}=-\omega_{\alpha b a}$ are the dreibein affinities and $R_{\alpha \beta a b} \equiv \partial_{\alpha} \omega_{\beta a b}+\omega_{\alpha a c} \omega_{\beta b}^{c}-(\beta \alpha)$ is the curvature tensor. For simplicity, we deal with the source-free case; matter coupling may be included in the standard way, and its effects will be noted. The ( $P$ - and $T$-nonconserving) mass terms are the ChernSimons expressions. They are closely related to the well-known four-dimensional $(\alpha=0,1,2,3)$ Pontryagin densities $X^{\alpha}$, defined by

$$
\begin{aligned}
& \boldsymbol{P}_{\boldsymbol{V}} \equiv \operatorname{tr}^{*} \boldsymbol{F}^{\alpha \beta} \boldsymbol{F}_{\alpha \beta} \equiv \partial_{\alpha} \boldsymbol{X}_{\boldsymbol{V}}{ }^{\alpha}, *^{*} \boldsymbol{F}^{\alpha \beta} \equiv \frac{1}{2} \epsilon^{\alpha \beta \lambda \sigma} \boldsymbol{F}_{\lambda \sigma} ; \\
& \boldsymbol{P}_{G} \equiv \operatorname{tr}^{*} R^{\alpha \beta}{ }_{a b} \boldsymbol{R}_{\alpha \beta}{ }^{a b} \equiv \partial_{\alpha} X_{G}{ }^{\alpha},{ }^{*} R^{\alpha \beta}{ }_{a b} \equiv \frac{1}{2} \epsilon^{\alpha \beta \lambda \sigma \sigma} \boldsymbol{R}_{\lambda \sigma a b} .
\end{aligned}
$$

The integrals of the $\alpha=3$ components, $X^{3}$, of the $\boldsymbol{X}^{\alpha}$ over the three-dimensional subspace excluding $x^{3}$, and with $x^{3}$ dependence suppressed, are (up to constants) just our $I_{\mathrm{cs}}$.

The source-free equations which follow from (1) are

$$
D_{\alpha} F^{\alpha \beta}+\mu * F^{\beta}=0, \quad * F^{\beta} \equiv \frac{1}{2} \epsilon^{\beta \lambda \sigma} F_{\lambda \sigma}
$$

and

$$
G^{\alpha \beta}+\mu^{-1} C^{\alpha \beta}=0, \quad G^{\alpha \beta} \equiv R^{\alpha \beta}-\frac{1}{2} g^{\alpha \beta} R, \quad C^{\alpha \beta} \equiv g^{-1 / 2} \epsilon^{\alpha \lambda \sigma} D_{\lambda}\left(\boldsymbol{R}_{\sigma}^{\beta}-\frac{1}{4} \delta_{\sigma}{ }^{\beta} R\right) .
$$


Despite the dreibein dependence of $I_{G C S}$, its variation is of purely metric nature, like that of the Einstein action; hence the metric form of (3b). We have made use here of the equivalence between Riemann and Ricci tensors in three dimensions, which also implies that flat space is the only solution of pure Einstein theory. Each term in (3) separately satsifies a covariant conservation identity. In three dimensions, $\boldsymbol{C}^{\mu \nu}$ is the conformal tensor replacing the normal Weyl tensor, which vanishes identical$1 \mathrm{y}^{4}$; the density $g^{1 / 2} \boldsymbol{C}_{\nu}{ }^{\mu}$ is invariant under conformal transformations of the metric. In addition to being conserved, $C^{\mu \nu}$ is symmetric by virtue of the usual Bianchi identity and manifestly traceless; the scalar curvature $\boldsymbol{R}$ therefore vanishes in the absence of sources.

To demonstrate the massive nature of the excitations, the dual of the vector equation (3a),

$$
O^{\alpha \beta}(\mu) * F_{B} \equiv\left(\mu g^{\alpha \beta}+\epsilon^{\alpha \beta} \gamma_{\gamma}\right) * F_{\beta}=0,
$$

and the gravitational ones,

$$
O_{\alpha \beta}^{\lambda \sigma}(\boldsymbol{\mu}) \boldsymbol{R}_{\lambda \sigma} \equiv\left[\left(\delta_{\alpha}{ }^{\lambda} \delta_{\beta}{ }^{\sigma}-\frac{1}{2} g_{\alpha \beta} g^{\lambda \sigma}\right)+\mu^{-1} g^{-1 / 2} \boldsymbol{\epsilon}_{\alpha}{ }^{\nu \tau}\left(\delta_{\boldsymbol{\tau}}{ }^{\lambda} \delta_{\beta}{ }^{\sigma}-\frac{1}{4} g^{\lambda \sigma} g_{\beta \tau}\right) D_{\nu}\right] \boldsymbol{R}_{\lambda \sigma}=0,
$$

may be iterated with $O(-\mu)$ to yield

$$
\left(D^{\alpha} D_{\alpha}+\mu^{2}\right) * F^{\nu}=\epsilon^{\nu \beta \gamma} \gamma\left[* F_{\beta}, * F_{\gamma}\right]
$$

and, using the $R=0$ property,

$$
\left(D^{\alpha} D_{\alpha}+\mu^{2}\right) R_{\beta \gamma}=3 R_{\beta}{ }^{\sigma} R_{\sigma \gamma}-g_{\beta \gamma} R_{\alpha \rho} R^{\alpha \rho} .
$$

In linearized approximation, which governs the kinematics of our theories, the right-hand sides are absent and we see that, whatever their number or spin, all particles are massive, with causal rather than tachyonic propagation. The latter fact is not controllable since the action depends on $\mu$ rather than $\boldsymbol{\mu}^{2}$, but may be understood in terms of the a priori positivity of the energy, and Bel-Robinson superenergy, densities of the theories. ${ }^{2}$

The nature of the excitations only becomes manifest by , more detailed analysis. The linearized actions are first expressed in terms of the Abelian gauge-invariant components of the fields. For the linearized Yang-Mills theory [ equal to the topologically massive Maxwell multiplet], these are the transverse components of the spatial vector potentials, $\boldsymbol{A}_{T}{ }^{i} \equiv \boldsymbol{\epsilon}^{i j} \hat{\partial}_{j} \varphi$, with $\hat{\partial}_{j} \equiv \partial_{j}\left(-\nabla^{2}\right)^{-1 / 2}$, and the longitudinal electric fields. The resulting constraint-free action is of Klein-Gordon form for $\varphi$; it describes one degree of freedom per color. For linearized gravity, we use the following three gaugeinvariant components of $h_{\mu \nu}=\kappa^{-1}\left(g_{\mu \nu}-\eta_{\mu \nu}\right)$ :

$$
\varphi=h^{i i}+\hat{\partial}_{i} \hat{\partial}_{j} h^{i j}, \quad \lambda=\nabla^{2} h^{00}+2 \partial_{i} \dot{h}^{0 i}-\hat{\partial}_{i} \hat{\partial}_{j} \ddot{h}_{i j}-\square \varphi, \quad \sigma=\epsilon^{i j}\left(\partial_{j} h^{0 i}-\hat{\partial}_{j} \hat{\partial}_{k} \dot{h}^{k i}\right) .
$$

In terms of these, the linearized action reads

$$
I_{G L}=-\frac{1}{2} \int d^{3} x\left[\left(\varphi \square \varphi+\sigma^{2}+\lambda \varphi\right)+\mu^{-1} \lambda \sigma\right] .
$$

Note that all triple time derivatives are safely hidden in the linearized Weyl-invariant Lagrange multiplier and constrained fields $(\lambda, \sigma)$ and that the Einstein (or topological) term alone is clearly vacuous. Elimination of $\lambda$ immediately leads to the unconstrained form

$$
I_{G L}=-\frac{1}{2} \int d^{3} x \varphi\left(\square+\mu^{2}\right) \varphi
$$

which is identical to the vector action, although the respective $\varphi$ variables have different Lorentz transformation properties. Note that $\varphi$ is essentially the conformal part of the metric, which appears with ghost sign in the conventional Einstein action. Accordingly, the overall sign of (7) is the physical one because the sign of the Einstein term in (1b) is opposite to that required in four dimensions.

Calculation of the linearized Poincare generators shows further that, despite the scalar form (7) of the action, the vector theory's particle has spin \pm 1 , while the graviton has spin \pm 2 . The spin sign is correlated to that of $\mu$ in the action. ${ }^{2}$ [In three dimensions spin is a rotational pseudoscalar and can have either sign. The value of the spin only emerges from study of the full $\mathrm{SO}(2,1)$ generators, and it is determined by the (invisible) temporal indices of the field variables.] As was already apparent from its topological origins or from the epsilon tensor, a single spin value reflects $T$ and $P$ nonconservation. [These discrete symmetries may be restored by considering a doublet of models and opposite mass terms.] Our excitation spectra differ discontinuously both from the massless ones in which the vector 
particle is spinless ${ }^{5}$ and the graviton is absent altogether, and from those of the traditional gauge-noncovariant massive models, which have two degrees of freedom for either case.

The topological terms in the Lagrangians change by the total divergences under gauge transformations; in the vector case, it is known that $I_{V C S}$ change by the winding number ${ }^{6} w(U)$ under "large" gauge transformations $U$ :

$$
\begin{aligned}
& A_{\mu} \rightarrow U^{-1} A_{\mu} U+U^{-1} \partial_{\mu} U, \\
& I_{V C S} \rightarrow I_{V C S}+8 \pi^{2} \mu g^{-2} w(U), \\
& w(U) \equiv\left(24 \pi^{2}\right)^{-1} \int d^{3} x \epsilon^{\alpha \beta \gamma} \operatorname{tr}\left[\partial_{\alpha} U\left(U^{-1} \partial_{\beta} U\right)\left(U^{-1} \partial_{\gamma} U\right) U^{-1}\right] .
\end{aligned}
$$

Therefore $\exp \left(i I_{V C S}\right)$ is gauge invariant only if the quantization condition

$$
\mu\left(g^{2} / 4 \pi\right)^{-1}=n, \quad n=0, \pm 1, \pm 2 \ldots,
$$

holds. Otherwise, the expectation value of a gauge-invariant operator $O$ would be undefined, as can be seen from the functional integral representation $\langle O\rangle=Z^{-1} \int D A O \exp \left(i I_{V}\right)$, with a gaugeinvariant measure $D A$ and normalization $Z$. Under a change of variables $A \rightarrow A^{u}$, where $A^{u}$ is a (large) gauge of transform of $A,\langle O\rangle$ would change by the phase implied by (8), which would only be unity if (9) held. ${ }^{7}$ The same result emerges in a Euclidean formulation since $I_{V C S}$ is independent of the metric's signature, but depends only on the nature of the maximal compact subgroup of the internal gauge group. The gravitational action's change under large local Lorentz transformations is formally identical, but as this "internal" group, $\mathrm{SO}(2,1)$, has the trivial compact subgroup SO(2) we cannot draw the same conclusion for $\mu \kappa^{2}$ in Minkowski signature. That is perhaps fortunate since that quantity may have to be infinitely renormalized in the quantum theory.

Quantization of the models is straightforward. For the vector theory, already superrenormalizable when $\mu=0$, the mass term acts as an infrared cutoff, since the propagator behaves as $\left(p^{2}\right.$ $+\mu p)^{-1}$. This gain of one power of momentum should suffice to cut of the low frequencies at each loop order. Explicit loop calculations of both Abelian and non-Abelian vector models coupled to fermions have been performed; although everything is finite, some of the finite results are actually regularization dependent. ${ }^{2}$

In gravity, there is a dramatic improvement in the ultraviolet behavior of the conformal sector of the theory, where the propagator now decays as $p^{-3}$. The model would be manifestly power-counting renormalizable [with $\kappa^{2} \mu$ as effective coupling constant] were it not for the scale factor, i.e., the part $\rho \eta_{\alpha \beta}$ of $h_{\alpha \beta}$ in the linearized approximation. This mode retains the conventional $p^{-2}$ propagation because the conformally invariant topolog- ical part does not affect it. ${ }^{8}$ There is a close parallel with four-dimensional theory when the conformal term $\sim R_{\mu \nu}^{2}-\frac{1}{3} R^{2}$ is added to the Einstein action. ${ }^{9}$ However, in four dimensions, ghosts are also introduced in the process. In either dimension, conformal invariance is removable by further addition of a term $\sim R^{2}$, but it is nonunitary.

It appears likely that supergravity can be constructed. When we adjoin to the $(P$ - and $T$-invariant) Rarita-Schwinger action $\frac{1}{2} i \int \epsilon^{\alpha \beta} \gamma_{\bar{\psi}}{ }_{\alpha} \partial_{\beta} \psi_{\gamma} d^{3} x$ (which, like the Einstein action, is trivial by itself), the supersymmetric companion of $X_{G}{ }^{3}$ derived from that of $* R R$ yields an action for a propagating massive spin $-\frac{3}{2}$ fermion. Existence of supergravity would also provide a simple proof of energy positivity of our full nonlinear gravity model. ${ }^{10}$

The external-source problem has also been analyzed. For gravity, the short-range behavior is exhibited by the effective Yukawa attraction, $H_{\text {in t }} \sim-\int T_{00}\left(-\nabla^{2}+\mu^{2}\right)^{-1} T_{00}$ of weak static $T_{00}$ sources. This contrasts with the interactions mediated by Einstein gravity (the $\mu \rightarrow \infty$ limit of our theory). There, the linearized propagator leads to a coupling $\sim \int d p p^{-2}\left[T_{\nu}{ }^{\mu}(p) T_{\mu}{ }^{\nu}(-p)\right.$ $\left.-T_{\mu}{ }^{\mu}(p) T_{\nu}{ }^{\nu}(-p)\right]$. Consequently, there is no $T^{00}-T^{00}$ term at all there, and the entire interaction is purely contact, since the $p^{-2}$ pole is canceled by the momenta implicit in $T^{\mu \nu}$ to ensure its conservation. Nevertheless the Newtonian component of the metric, defined as usual from the linearized part of $G^{00}$, still behaves asymptotically as $\nabla^{-2} T^{00}$, and the total energy is expressed by the same flux integral at spatial infinity as in ordinary gravity. The vector theory also implies short-range source interactions, but differs from the massless case in that the longitudinal electric field becomes short range and the total charge is read off asymptotically from the magnetic potential. [In both cases, it is the lowest derivative term in the constraint equations which carries the asymptotic information about the "charges."] 
A number of questions are raised by these results. First, it would be of interest to study in more detail the infrared properties of the vector model to verify that the mass provides a cutoff beyond one loop. Second, investigation of the ultraviolet behavior of gravity and supergravity should reveal the extent of the improvement due to the conformal sector's asymptotics. This could also be done by determining the form of the (space-nonlocal) self-interaction $V(\Phi)$ in the full nonlinear action, $I_{G}=-\frac{1}{2} \int d x\left[\Phi\left(\square+\mu^{2}\right) \Phi+V(\Phi)\right]$, expressed in terms of the single dynamical variable $\Phi$.

The most relevant problem is clearly the relation of these models to four-dimensional theory. Although the normal terms (taken in Euclidean signature) can be considered as high-temperature limits, ${ }^{11}$ or dimensional reductions of the latter, the topological terms have a different origin, in the $\theta$ vacuum of the four-dimensional physics. ${ }^{12}$ However, since $\theta$ terms are generally present in four dimensions, naturalness suggests that the corresponding topological terms be included in the high-temperature limit.

Similar topologically augmented models could also be introduced in other odd-dimensional systems. However, as is clear from their origin, the topological parts will be of higher derivative order and powers of the fields, unless expressed in terms of higher rank gauge fields. Such variables and terms have arisen naturally in higherdimensional supergravity and may perhaps provide a way of introducing mass without Higgs scalars.

Details of this work will be reported elsewhere. ${ }^{2}$

This work is supported in part by National Science Foundation Grant No. Phy 78-09644 A02 and by U. S. Department of Energy Contract No. DE AC02-76ER03069.

${ }^{1}$ Gauge-invariant mass terms for vector gauge theor- ies were introduced by R. Jackiw and S. Templeton, Phys. Rev. D 23, 2291 (1981); J. Schonfeld, Nucl. Phys. B185, 157 (1981). See also W. Siegel, Nucl. Phys. $\overline{\mathrm{B} 156}, 135$ (1979). More detailed analysis, summarized in this Letter, is given by S. Deser, R. Jackiw, and S. Templeton, to be published.

${ }^{2}$ Deser, Jackiw, and Templeton, Ref. 1.

${ }^{3}$ For a description, see S. S. Chern, Complex Manifolds without Potential Theory (Springer, Berlin, 1979), 2nd ed.

${ }^{4}$ L. P. Eisenhart, Riemannian Geometry (Princeton Univ. Press, Princeton, N.J., 1949); R. Arnowitt, S. Deser, and C. W. Misner, in Gravitation, edited by L. Witten (Wiley, New York, 1962); J. W. York, Phys. Rev. Lett。26, 1656 (1971).

${ }^{5}$ B. Binegar, to be published.

${ }^{6}$ R. Jackiw and C. Rebbi, Phys. Rev. Lett. 37, 172 (1976); R. Jackiw, Rev. Mod. Phys. 52, 661 (1980).

${ }^{7} \mathrm{An}$ alternative argument is based on the fact that the phase exponential changes by $f(N) \equiv \exp \left[2 \pi i \mu\left(g^{2} / 4 \pi\right)^{-1} N\right]$ as the integration (without change of variable) ranges over gauge copies, with relative winding number $N$, of a given configuration. Without quantization, the sum of these contributions is the overall factor $\Sigma_{N} f(N)$, which vanishes, rather than $\sim \Sigma_{N} 1$ which is harmlessly canceled by $Z$. However, J. Schonfeld private communication) suggests that the "zero" could perhaps similarly be canceled by $Z$; whether this is acceptable is not clear to us.

${ }^{8}$ We thank E. Witten for pointing out an error in an earlier version of this argument.

${ }^{9}$ K. S. Stelle, Phys. Rev. D 16, 953 (1977).

${ }^{10} \mathrm{~S}$. Deser and C. Teitelboim, Phys. Rev. Lett. 39, 249 (1977); M. Grisaru, Phys. Lett. 73B, 207 (1978); E. Witten, Commun. Math. Phys. $80, \overrightarrow{381}$ (1981).

${ }^{11} \mathrm{~S}$. Weinberg, in Understanding the Fundamental Interactions, edited by A. Zichichi (Plenum, New York, 1978); A. Linde, Rep. Prog. Phys. 42,389 (1979); D. J. Gross, R. D. Pisarski, and L. G. Yaffe, Rev. Mod. Phys. 53, 43 (1981). For gravity, however, the sign taken here for the Einstein term is opposite to that in the original four-dimensional theory.

${ }^{12}$ For vector theories, see Ref. 6; for gravity theories, see S. Deser, M. Duff, and C. Isham, Phys. Lett. $\underline{93 B}_{9}$ 419 (1980); C. Isham, International Center for Theoretical Physics Report No. 81/82-1 (to be published). 